\title{
New limits on hidden photons from past electron beam dumps
}

\author{
Sarah Andreas, ${ }^{*}$ Carsten Niebuhr, ${ }^{\dagger}$ and Andreas Ringwald ${ }^{*}$ \\ Deutsches Elektronen-Synchrotron DESY, 22607 Hamburg, Notkestrasse 85, Germany
}

(Received 2 October 2012; published 16 November 2012)

\begin{abstract}
Hidden sectors with light extra U(1) gauge bosons, so-called hidden photons, have recently attracted some attention because they are a common feature of physics beyond the Standard Model like string theory and supersymmetry and additionally are phenomenologically of great interest regarding recent astrophysical observations. The hidden photon is already constrained by various laboratory experiments and presently searched for in running as well as upcoming experiments. We summarize the current status of limits on hidden photons from past electron beam dump experiments including two new limits from such experiments at the High Energy Accelerator Research Organization in Japan (KEK) and the Laboratoire de l'accelérateur linéaire (LAL, Orsay) that have so far not been considered. All our limits take into account the experimental acceptances obtained from Monte Carlo simulations.
\end{abstract}

DOI: 10.1103/PhysRevD.86.095019

PACS numbers: 12.60.Cn, 14.70.Pw, 13.85.Rm

\section{MOTIVATION}

Light extra $\mathrm{U}(1)$ gauge bosons appear very naturally in well-motivated extensions of the standard model (SM). Even if the paradigm of a highly symmetric high energy theory is to unify particles into representations of a largerank local gauge group, the phenomenological fact that at low energies these large gauge symmetries are seemingly broken possibly leaves us with the existence of many lower rank symmetries. In particular U(1)s are potentially most numerous since they are the lowest-rank local symmetries. Moreover, some of these U(1)s may be hidden, because the SM particles are not charged under them, and therefore escaped detection until now. Most notably, such hidden sectors often occur in supersymmetric extensions or superstring embeddings of the SM [1-5].

On general grounds, the dominant interaction of hidden $\mathrm{U}(1)$ gauge bosons $\gamma^{\prime}$ (hidden sector photons, short hidden photons) with the SM at low energies generically appears already at the dimension four level through kinetic mixing with the ordinary photon [6]. Correspondingly, the leading terms of the low energy effective Lagrangian of the minimal hidden U(1) extension of the SM read,

$$
\mathcal{L}_{\mathrm{eff}}=\mathcal{L}_{\mathrm{SM}}-\frac{1}{4} F_{\mu \nu}^{\prime} F^{\prime \mu \nu}+\frac{m_{\gamma^{\prime}}^{2}}{2} A_{\mu}^{\prime} A^{\prime \mu}-\frac{\chi}{2} F_{\mu \nu}^{\prime} F^{\mu \nu},
$$

where $F_{\mu \nu}^{\prime}$ is the field strength of the hidden gauge field $A_{\mu}^{\prime}, m_{\gamma^{\prime}}$ the mass of the hidden photon, and $F_{\mu \nu}$ the field strength of the ordinary electromagnetic gauge field.

The hidden photon mass $m_{\gamma^{\prime}}$ and the kinetic mixing parameter $\chi$ have to be determined either theoretically, by specifying an ultraviolet completion of the theory, or phenomenologically, by comparing with observations.

\footnotetext{
*sarah.andreas@desy.de

$\dagger$ carsten.niebuhr@desy.de

†andreas.ringwald@desy.de
}

In this context, two very interesting mass regions have been identified recently:

(i) $m_{\gamma^{\prime}} \sim$ meV: Hidden photons in this mass range may explain the $\sim 2 \sigma$ excess of dark radiation in the universe [7], beyond the one from ordinary photons and neutrinos, reported by recent global cosmological analyses $[8,9]$. This possibility can be tested decisively in the next generation of light-shiningthrough-a-wall experiments [10].

(ii) $m_{\gamma^{\prime}} \sim \mathrm{GeV}$ : Hidden photons in this mass range may explain the observed $\sim 3 \sigma$ deviation of the anomalous magnetic moment of the muon [11] from the value expected in the SM [12]. Moreover, they might explain possible terrestrial and cosmic ray dark matter anomalies-notably the possible direct detection of dark matter by DAMA [13], CoGeNT [14,15] and CRESST [16,17], in contrast to its nonobservation in CDMS [18] and XENON [19], and the observations of an excess in cosmic ray electrons and/or positrons observed by PAMELA [20] and Fermi [21]-if dark matter resides in the hidden sector too and is charged under the hidden U(1) [22-28]. This possibility can be tested seriously with new accelerator based experiments [29-31], especially with new beam dump and fixed target experiments exploiting high intensity electron $[32,33]$ and proton beams $[34,35]$.

Motivated by this strong physics case, a number of electron beam dump and fixed target experiments to search for $\mathrm{GeV}$ scale hidden photons (dark forces) have been proposed or even taken first data [36-38]. In this context it is very important to analyze also results from past electron beam dump experiments in terms of hidden photons-a task which was accomplished quite exhaustively in Ref. [32]. However, in this paper two experiments have not been considered: (i) a beam dump experiment searching for neutral penetrating particles exploiting an electron linear accelerator at the High Energy Accelerator Research Organization (KEK) [39] and (ii) a beam dump 
experiment exploiting the Orsay Linac originally analyzed in terms of production and late decay of scalars (Higgs) and pseudoscalars (axions) [40]. ${ }^{1}$ In this paper, we derive the corresponding bounds on hidden photons, which exceed the bounds previously established by other electron beam dump experiments in a certain region of the parameter space.

\section{II. $\gamma^{\prime}$ IN ELECTRON BEAM DUMPS}

In this section, we summarize the relevant formula (based on Ref. [32]) and computational steps necessary to determine the expected signatures of a hidden photon $\gamma^{\prime}$ in a beam dump experiment and to deduce the limits on its mass $m_{\gamma^{\prime}}$ and kinetic mixing $\chi$ set by different experiments.

\section{A. $\gamma^{\prime}$ production in bremsstrahlung}

Hidden photons are generated in electron (or positron) collisions on a fixed target by a process analogous to ordinary photon bremsstrahlung. For an incoming electron with energy $E_{e}$ the corresponding differential cross section in the range

$$
m_{e} \ll m_{\gamma^{\prime}} \ll E_{e} \quad \text { and } \quad x_{e} \theta_{\gamma^{\prime}}^{2} \ll 1
$$

is given in Ref. [32] Eq. (A12) by

$$
\begin{aligned}
\frac{d \sigma}{d x_{e} d \cos \theta_{\gamma^{\prime}}}= & 8 \alpha^{3} \chi^{2} E_{e}^{2} x_{e} \xi \sqrt{1-\frac{m_{\gamma^{\prime}}^{2}}{E_{e}^{2}}}\left[\frac{1-x_{e}+\frac{x_{e}^{2}}{2}}{U^{2}}\right. \\
& \left.+\frac{\left(1-x_{e}\right)^{2} m_{\gamma^{\prime}}^{4}}{U^{4}}-\frac{\left(1-x_{e}\right) x_{e} m_{\gamma^{\prime}}^{2}}{U^{3}}\right]
\end{aligned}
$$

where $x_{e}=E_{\gamma^{\prime}} / E_{e}$ is the fraction of the incoming electron's energy carried by the hidden photon, $\theta_{\gamma^{\prime}}$ is the lab frame angle between emitted $\gamma^{\prime}$ and incoming electron and $Z$ and $A$ are atomic number and mass number of the nucleus in the target. The effective flux of photons $\xi$ is given by

$$
\xi\left(E_{e}, m_{\gamma^{\prime}}, Z, A\right)=\int_{t_{\min }}^{t_{\max }} d t \frac{t-t_{\min }}{t^{2}} G_{2}(t),
$$

where $t_{\min }=\left(m_{\gamma^{\prime}}^{2} / 2 E_{e}\right)^{2}, t_{\max }=m_{\gamma^{\prime}}^{2}$ and the electric form factor $G_{2}(t)$ defined in Ref. [32] consists of an elastic and an inelastic contribution both of which depend on the atomic number $Z$ and mass $A$. The function $U$ describes the virtuality of the intermediate electron in initial-state bremsstrahlung and is given by

$$
U\left(x_{e}, E_{e}, m_{\gamma^{\prime}}, \theta_{\gamma^{\prime}}\right)=E_{e}^{2} x_{e} \theta_{\gamma^{\prime}}^{2}+m_{\gamma^{\prime}}^{2} \frac{1-x_{e}}{x_{e}}+m_{e}^{2} x_{e} .
$$

Integrating Eq. (2) over the emission angle $\theta_{\gamma^{\prime}}$ of the hidden photon from 0 to some maximum angle $\theta_{\max }$ set by the

\footnotetext{
${ }^{1}$ In Ref. [41] it had already been suggested that the electron beam dump experiment at Orsay could be used to constrain the more general $U$-boson for which another limit from proton beam dumps was obtained in Ref. [42].
}

geometry of the experiment (for the experiments under consideration $\theta_{\max }<0.5 \mathrm{rad}$ ) we obtain ${ }^{2}$

$$
\frac{d \sigma}{d x_{e}}=4 \alpha^{3} \chi^{2} \xi \sqrt{1-\frac{m_{\gamma^{\prime}}^{2}}{E_{e}^{2}}} \frac{1-x_{e}+\frac{x_{e}^{2}}{3}}{m_{\gamma^{\prime}}^{2} \frac{1-x_{e}}{x_{e}}+m_{e}^{2} x_{e}} .
$$

\section{B. Number of expected events behind a beam dump}

For a beam dump experiment with an electron beam of energy $E_{0}$ incident on a target (cf. Fig. 1) one has to take into account that the initial energy of the electrons in the beam becomes degraded as they pass through the target and interact with the material. This is described by the energy distribution of the electrons after passing through a medium of $t$ radiation length which according to Tsai [43] is roughly given by

$$
I_{e}\left(E_{0}, E_{e}, t\right)=\frac{1}{E_{0}} \frac{\left[\ln \left(\frac{E_{0}}{E_{e}}\right)\right]^{b t-1}}{\Gamma(b t)},
$$

where $E_{0}$ is the initial monochromatic electron beam energy at $t=0, \Gamma$ is the Gamma function and $b=\frac{4}{3}$. The bremsstrahlung cross section from the previous subsection which depends on the energy $E_{e}$ of the electrons therefore has to be convoluted with this energy distribution and integrated over the length $L_{\mathrm{sh}}$ of the target plus shield. Together with Eq. (6), the total number of hidden photons with an energy $E_{\gamma^{\prime}} \equiv x_{0} E_{0}$ that are produced in the target via bremsstrahlung off the electron beam and that decay at a distance $z$ behind the front edge of the target is then given by

$$
\begin{aligned}
\frac{d N}{d x_{0} d z}= & N_{e} \frac{N_{0} X_{0}}{A} \int_{E_{\gamma^{\prime}}+m_{e}}^{E_{0}} d E_{e} \int_{0}^{T} d t\left[I_{e}\left(E_{0}, E_{e}, t\right)\right. \\
& \left.\times\left.\frac{E_{0}}{E_{e}} \frac{d \sigma}{d x_{e}}\right|_{x_{e}=\frac{E_{\gamma^{\prime}}}{E_{e}}} \frac{d P\left(z-\frac{X_{0}}{\rho} t\right)}{d z}\right],
\end{aligned}
$$

where $N_{e}$ and $E_{0}$ are the number and energy of the incident electrons, respectively, $N_{0} \simeq 6 \times 10^{23} \mathrm{~mole}^{-1}$ is Avogadro's number, $\rho\left[\mathrm{g} / \mathrm{cm}^{3}\right]$ and $X_{0}\left[\mathrm{~g} / \mathrm{cm}^{2}\right]$ are the density and unit radiation length of the target material, respectively, and $T \equiv \rho L_{\mathrm{sh}} / X_{0}$ is the length $L_{\mathrm{sh}}$ of target plus shield in units of radiation length. The differential cross section $d \sigma / d x_{e}$ discussed in Sec. II A is given in Eq. (5). The differential decay probability $d P / d z$ is defined as

$$
\frac{d P(l)}{d l}=\frac{1}{l_{\gamma^{\prime}}} e^{-l / l_{\gamma^{\prime}}}
$$

where $l_{\gamma^{\prime}}$ is the decay length of the hidden photon $l_{\gamma^{\prime}}=$ $\gamma \tau_{\gamma^{\prime}}=\frac{E_{\gamma^{\prime}}}{m_{\gamma^{\prime}}} \frac{1}{\Gamma_{\gamma^{\prime}}}$. For the mass range of interest, the total decay width $\Gamma_{\gamma^{\prime}}$ is given by

\footnotetext{
${ }^{2}$ Note that this expression includes a factor $1 / 2$ which has erroneously been omitted in Ref. [32].
} 


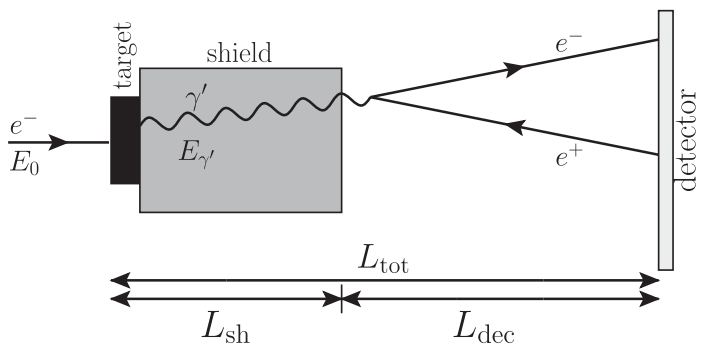

FIG. 1. Sketch of the setup of an electron beam dump experiment illustrating the definitions of the lengths $L_{\mathrm{sh}}, L_{\mathrm{dec}}$ and $L_{\mathrm{tot}}$ used in the text. An incoming electron beam of energy $E_{0}$ hits the target and produces in bremsstrahlung a hidden photon $\gamma^{\prime}$ with energy $E_{\gamma^{\prime}}$ that decays behind the shield e.g., into $e^{+} e^{-}$which can then be observed in the detector.

$$
\Gamma_{\gamma^{\prime}}=\Gamma_{\gamma^{\prime} \rightarrow e^{+} e^{-}}+\Gamma_{\gamma^{\prime} \rightarrow \mu^{+} \mu^{-}}\left[1+R\left(m_{\gamma^{\prime}}\right)\right],
$$

where the second term is only present for $m_{\gamma^{\prime}} \geq 2 m_{\mu}$, the partial decay width into leptons is given by [12]

$$
\Gamma_{\gamma^{\prime} \rightarrow l^{+} l^{-}}=\frac{\alpha \chi^{2}}{3} m_{\gamma^{\prime}}\left(1+2 \frac{m_{l}^{2}}{m_{\gamma^{\prime}}^{2}}\right) \sqrt{1-4 \frac{m_{l}^{2}}{m_{\gamma^{\prime}}^{2}}},
$$

and $R(\sqrt{s})$ is defined as the energy dependent ratio $\frac{\sigma\left(e^{+} e^{-} \rightarrow \text { hadrons, } \sqrt{s}\right)}{\sigma\left(e^{+} e^{-} \rightarrow \mu^{+} \mu^{-}, \sqrt{s}\right)}$ taken from Ref. [44].

\section{Special case: Thick target beam dump experiment}

In the case of a thick target experiment, which we are interested in, most of the hidden photon production takes place within the first radiation length so that the $t$ dependence in the $\gamma^{\prime}$ decay probability can be neglected and Eq. (7) simplifies to

$$
\begin{aligned}
\frac{d N}{d x_{0} d z} \simeq & N_{e} \frac{N_{0} X_{0}}{A} \int_{E_{\gamma^{\prime}}+m_{e}}^{E_{0}} d E_{e} \int_{0}^{T} d t\left[I_{e}\left(E_{0}, E_{e}, t\right)\right. \\
& \left.\times\left.\frac{E_{0}}{E_{e}} \frac{d \sigma}{d x_{e}}\right|_{x_{e}=\frac{E_{\gamma^{\prime}}}{E_{e}}} \frac{d P(z)}{d z}\right] .
\end{aligned}
$$

After carrying out the integration over $z$ from $L_{\mathrm{sh}}$ to $L_{\mathrm{tot}} \equiv$ $L_{\mathrm{sh}}+L_{\mathrm{dec}}$, where $L_{\mathrm{sh}}$ is the length of target plus shield and $L_{\mathrm{dec}}$ the length of the decay region, as sketched in Fig. 1, this becomes

$$
\begin{aligned}
\frac{d N}{d x_{0}} \simeq & N_{e} \frac{N_{0} X_{0}}{A} \int_{E_{\gamma^{\prime}}+m_{e}}^{E_{0}} d E_{e} \int_{0}^{T} d t\left[I_{e}\left(E_{0}, E_{e}, t\right)\right. \\
& \times\left.\frac{E_{0}}{E_{e}} \frac{d \sigma}{d x_{e}}\right|_{x_{e}=\frac{E_{\gamma^{\prime}}}{E_{e}}} e^{-L_{\mathrm{sh}} / l_{\gamma^{\prime}}}\left(1-e^{\left.-L_{\mathrm{dec}} / l_{\gamma^{\prime}}\right)}\right] .
\end{aligned}
$$

The total number of events behind the dump resulting from the decay of the hidden photon is then given by

$$
\begin{aligned}
N \simeq & N_{e} \frac{N_{0} X_{0}}{A} \int_{m_{\gamma^{\prime}}}^{E_{0}-m_{e}} d E_{\gamma^{\prime}} \int_{E_{\gamma^{\prime}}+m_{e}}^{E_{0}} d E_{e} \int_{0}^{T} d t \\
& \times\left[\left.I_{e}\left(E_{0}, E_{e}, t\right) \frac{1}{E_{e}} \frac{d \sigma}{d x_{e}}\right|_{x_{e}=\frac{E_{\gamma^{\prime}}}{E_{e}}}\right. \\
& \left.\times e^{-L_{\mathrm{sh}} / l_{\gamma^{\prime}}}\left(1-e^{-L_{\mathrm{dec}} / l_{\gamma^{\prime}}}\right)\right] \mathrm{BR}_{\mathrm{detect}},
\end{aligned}
$$

where $\mathrm{BR}_{\text {detect }}$ is the branching ratio into those decay products that the detector is sensitive to, i.e., electrons or muons or both.

\section{Acceptance of different experiments}

Up to now we have not taken into account that depending on the angle under which the final decay products are emitted and the geometry of the detector, not all events computed according to Eq. (13) are actually seen by the detector. With the use of MADGRAPH $[45,46]$ we generated for the different experiments (see Table I) Monte Carlo simulations of the hidden photon's production in bremsstrahlung followed by its decay into $e^{+} e^{-}$. Comparing the thereby obtained decay angles with the geometrical setup of the experiment an acceptance specific for each experiment can be determined. Repeating this procedure for every experiment along the rough exclusion contour obtained using Eq. (13) we can rescale the limit with the proper acceptance to get the final exclusion region. In the cases where the acceptances have been given in the experiment's paper, we compared and found them in reasonable agreement with the results of our Monte Carlo simulations.

\section{ELECTRON BEAM DUMP EXPERIMENTS}

An overview of the different electron beam dump experiments and their properties is shown in Table I.

TABLE I. Overview of the different beam dump experiments analyzed in this work and their specifications. The number of observed events $N_{\text {obs }}$ have directly been extracted from the experiment's papers and differ in the case of E141 and E137 slightly from the estimates used in Ref. [32] as do the corresponding 95\% C.L. values.

\begin{tabular}{lcccccrr}
\hline \hline Experiment & \multicolumn{1}{c}{$\begin{array}{c}E_{0} \\
{[\mathrm{GeV}]}\end{array}$} & electrons & Coulomb & $\begin{array}{c}L_{\text {sh }} \\
{[\mathrm{m}]}\end{array}$ & $\begin{array}{c}L_{\text {dec }} \\
{[\mathrm{m}]}\end{array}$ & $N_{\text {obs }}$ & \multicolumn{1}{c}{$N_{95 \% \text { up }}$} \\
\hline E141 [47] & $\mathrm{W}$ & 9 & $2 \times 10^{15}$ & $0.32 \mathrm{mC}$ & 0.12 & 35 & $1126_{-1126}^{+1312}$ \\
E137 [48] & $\mathrm{Al}$ & 20 & $1.87 \times 10^{20}$ & $30 \mathrm{C}$ & 179 & 204 & 0 \\
E774 [49] & $\mathrm{W}$ & 275 & $5.2 \times 10^{9}$ & $0.83 \mathrm{nC}$ & 0.3 & 2 & 3 \\
KEK [39] & $\mathrm{W}$ & 2.5 & $1.69 \times 10^{17}$ & $27 \mathrm{mC}$ & 2.4 & 2.2 & 0 \\
Orsay [40] & $\mathrm{W}$ & 1.6 & $2 \times 10^{16}$ & $3.2 \mathrm{mC}$ & 1 & 2 & 0 \\
\hline \hline
\end{tabular}


In Ref. [32], the limits set by the E141 and E137 experiments at the Stanford Linear Accelerator Center (SLAC) as well as the Fermilab E774 experiment have already been analyzed. In the present paper, we extend their analysis by two experiments that so far have not been considered: one electron beam dump experiment at KEK in Japan [39] and one at the Orsay Linac in France [40]. In addition, our analysis includes the experimental acceptances obtained from Monte Carlo simulations with MADGRAPH in the determination of the limits for all experiments, as described in Sec. IID.

For the SLAC E141 experiment [47], we extract from Fig. 1c for $x \geq 0.7$ a total of $1126 \pm 1312$ events, which corresponds to a 95\% C.L. upper limit of $N_{95 \% \text { up }}=3419$ events. The appropriate exclusion contour shown in Fig. 2 takes into account the acceptance from MADGRAPH.

As the SLAC E137 experiment reported in Ref. [48] that no candidate events were observed in their search for axionlike particles, the $95 \%$ C.L. upper limit is given by $N_{95 \% \text { up }}=3$ events. Together with the acceptance we then find the exclusion contour presented in Fig. 2.

For the Fermilab E774 experiment we find a total of zero events with excess multiplicity 2 from Fig. 4c in Ref. [49]. Resulting from a substraction of the background from the original spectrum, the statistical error of $\sqrt{89}$ is dominated by the total number of events in Fig. 4b. The acceptance corrected $95 \%$ C.L. upper limit of $N_{95 \% \text { up }}=18$ events leads to the exclusion contour in Fig. 2.

In the electron beam dump experiment at KEK [39] no signal was observed in their search for axionlike particles.

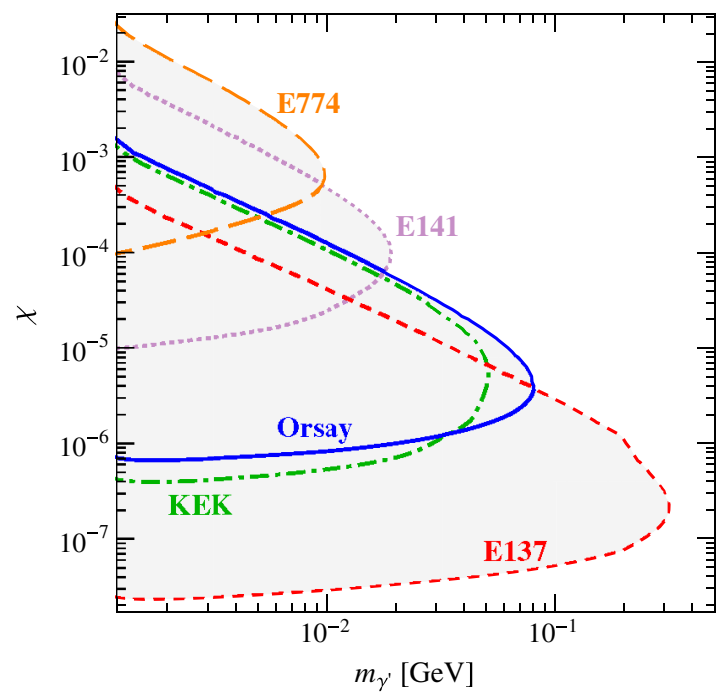

FIG. 2 (color online). Limits on the hidden photon mass $m_{\gamma^{\prime}}$ and kinetic mixing $\chi$ from different electron beam dump experiments. In addition to the limits from E141 (magenta dotted line), E137 (red dashed line) and E774 (orange long-dashed line) presented already in Ref. [32], the regions labeled KEK (green dash-dotted line) and Orsay (blue solid line) have been excluded in the present work.
The corresponding $95 \%$ C.L. upper limit $N_{95 \% \text { up }}$ of three events together with the acceptance leads for hidden photons to the exclusion contour presented in Fig. 2.

The electron beam dump experiment in Orsay [40] also found no positive signal when looking for light Higgs bosons. This translates to a $95 \%$ C.L. upper limit $N_{95 \% \text { up }}$ of three events on hidden photons. Considering the experiment's acceptance we find the exclusion contour shown in Fig. 2.

\section{DISCUSSION}

As presented in Fig. 2, the experiments at KEK and in Orsay were found to exclude a similar region of the parameter space which so far has not been constrained by any other electron beam dump experiment. Our limits from the previously analyzed experiments at SLAC and Fermilab are comparable to those derived in Ref. [32] but are generally slightly weaker because of the factor $1 / 2$ discrepancy in Eq. (5), the fact that our Monte Carlo simulations yield somewhat different experimental acceptances and due to a little different numbers of events $N_{95 \%}$ up used for our $95 \%$ C.L. contours.

Besides the limits from electron beam dump experiments discussed in this article, there are various other constraints on the hidden photon mass and kinetic mixing which we briefly summarize in the following. Their comparison to the electron beam dump experiments from Fig. 2 is shown in Fig. 3.

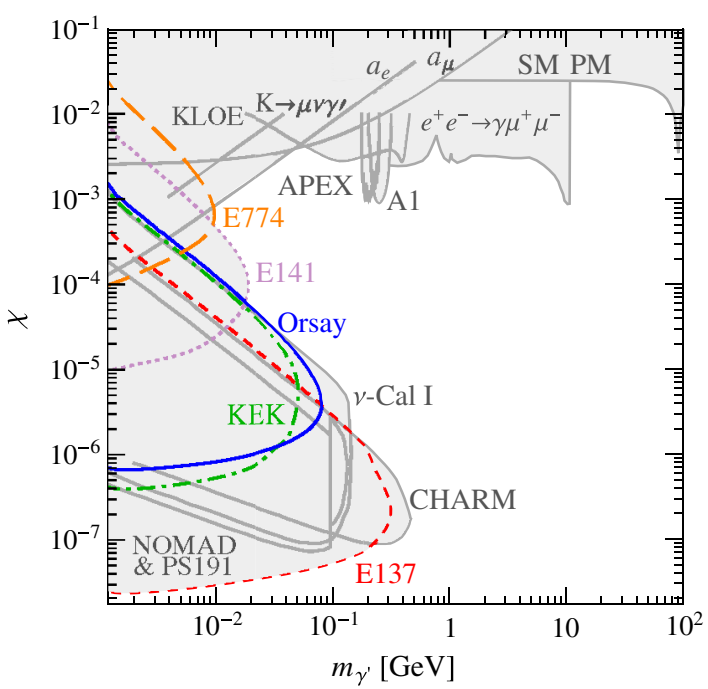

FIG. 3 (color online). Collection of all current limits on hidden photons: from the electron beam dump experiments of the present work (colored lines, cf. Fig. 2, all other limits as gray lines), Standard Model precision measurements, muon and electron anomalous magnetic moment, a reinterpretation of the BABAR search $e^{+} e^{-} \rightarrow \gamma \mu^{+} \mu^{-}$for pseudoscalars, the electron fixed target experiments A1 and APEX, the $\nu$-Cal I experiment at the Serpukhov proton beam dump, the KLOE experiment, the neutrino experiments NOMAD, PS191 and CHARM, and from the Kaon decay $K \rightarrow \mu \nu \gamma^{\prime}$, cf., text for details. 
In Ref. [50], SM precision measurements were found to exclude large values of hidden photon mass and kinetic mixing as indicated in Fig. 3 by the label $S M P M$. Furthermore, as presented in Ref. [12] the muon and electron anomalous magnetic moment receiving a oneloop contribution from the hidden photon place additional constraints labeled " $a_{\mu}$ " and " $a_{e}$ " respectively; the latter was updated [51,52] while this work has been completed. The reinterpretation of the BABAR search for a pseudoscalar around the $\Upsilon(3 S)$ resonance [53] in the process $e^{+} e^{-} \rightarrow \gamma \mu^{+} \mu^{-}$was used in Refs. [31,36,50,54] to derive a limit on hidden photons. The two fixed target experiments A1 at MAMI in Mainz [37] and APEX at JLab [38] both searching for hidden photons behind a thin target from bremsstrahlung off an electron beam started recently and were already able to set first new limits. Reanalyzing proton beam dump data from the $\nu$-Cal I experiment at the U70 accelerator at IHEP Serpukhov a region overlapping with the one of KEK and Orsay has been excluded in Ref. [55]. The KLOE-2 experiment [56] at the Frascati DA $\phi$ NE $\phi$-factory uses $e^{+} e^{-}$collisions to place further constraints. Very recently the production of hidden photons in the radiative decays of neutral pseudoscalar mesons, generated by a proton beam in neutrino experiments at CERN, has been constrained with NOMAD and PS191
[57] in the decay of $\pi^{0}$ and CHARM [58] in the one of $\eta$ and $\eta^{\prime}$. While this work was completed a new limit from the Kaon decay $K \rightarrow \mu \nu \gamma^{\prime}$ was derived [59] which would have improved the previous $a_{e}$ bound but is not competitive with the updated one.

An up-to-date overview of all current constraints on the mass $m_{\gamma^{\prime}}$ and kinetic mixing $\chi$ of the hidden photon from various searches including the electron beam dump experiments presented in this work is shown in Fig. 3. Despite the large number of constraints a broad region of the parameter space remains open and is partly going to be tested in currently already running [36-38] and planned future experiments [33,60,61], see Ref. [62] for an overview.

\section{ACKNOWLEDGMENTS}

We would like to thank Yuri Soloviev for pointing out Ref. [39] and Jonathan Jacobsohn for discussions at an early stage of this work. We are also grateful that Rouven Essig, Philip Schuster and Natalia Toro provided us with their MADGRAPH code for hidden photons which allowed us to simulate the acceptances of the different beam dump experiments. Special thanks to Rouven Essig for helpful discussions regarding the factor $1 / 2$ discrepancy in the cross section.
[1] S. A. Abel, M. D. Goodsell, J. Jaeckel, V. V. Khoze, and A. Ringwald, J. High Energy Phys. 07 (2008) 124.

[2] M. Goodsell, J. Jaeckel, J. Redondo, and A. Ringwald, J. High Energy Phys. 11 (2009) 027.

[3] M. Goodsell and A. Ringwald, Fortschr. Phys. 58, 716 (2010).

[4] M. Cicoli, M. Goodsell, J. Jaeckel, and A. Ringwald, J. High Energy Phys. 07 (2011) 114.

[5] M. Williams, C. P. Burgess, A. Maharana, and F. Quevedo, J. High Energy Phys. 08 (2011) 106.

[6] B. Holdom, Phys. Lett. 166B, 196 (1986).

[7] J. Jaeckel, J. Redondo, and A. Ringwald, Phys. Rev. Lett. 101, 131801 (2008).

[8] E. Komatsu et al. (WMAP Collaboration), Astrophys. J. Suppl. Ser. 192, 18 (2011).

[9] J. Dunkley et al., Astrophys. J. 739, 52 (2011).

[10] J. Redondo and A. Ringwald, Contemp. Phys. 52, 211 (2011).

[11] G. W. Bennett et al. (Muon G-2 Collaboration), Phys. Rev. D 73, 072003 (2006).

[12] M. Pospelov, Phys. Rev. D 80, 095002 (2009).

[13] R. Bernabei et al. (DAMA Collaboration), Eur. Phys. J. C 56, 333 (2008).

[14] C. E. Aalseth et al. (CoGeNT Collaboration), Phys. Rev. Lett. 106, 131301 (2011).

[15] C. E. Aalseth et al. (CoGeNT Collaboration), Phys. Rev. Lett. 107, 141301 (2011).
[16] G. Angloher et al., Eur. Phys. J. C 72, 1971 (2012).

[17] L. Stodolsky et al., J. Phys. Conf. Ser. 384, 012013 (2012).

[18] Z. Ahmed et al. (The CDMS-II Collaboration), Science 327, 1619 (2010).

[19] E. Aprile et al. (XENON100 Collaboration), Phys. Rev. Lett. 107, 131302 (2011).

[20] O. Adriani et al. (PAMELA Collaboration), Nature (London) 458, 607 (2009).

[21] A. A. Abdo et al. (The Fermi LAT Collaboration), Phys. Rev. Lett. 102, 181101 (2009).

[22] N. Arkani-Hamed, D. P. Finkbeiner, T. R. Slatyer, and N. Weiner, Phys. Rev. D 79, 015014 (2009).

[23] M. Pospelov and A. Ritz, Phys. Lett. B 671, 391 (2009).

[24] D. Feldman, Z. Liu, and P. Nath, Phys. Rev. D 79, 063509 (2009).

[25] D.E. Morrissey, D. Poland, and K. M. Zurek, J. High Energy Phys. 07 (2009) 050.

[26] Y. Mambrini, J. Cosmol. Astropart. Phys. 09 (2010) 022.

[27] Y. Mambrini, J. Cosmol. Astropart. Phys. 07 (2011) 009.

[28] S. Andreas, M.D. Goodsell, and A. Ringwald, arXiv:1109.2869.

[29] B. Batell, M. Pospelov, and A. Ritz, Phys. Rev. D 79, 115008 (2009).

[30] R. Essig, P. Schuster, and N. Toro, Phys. Rev. D 80, 015003 (2009).

[31] M. Reece and L.-T. Wang, J. High Energy Phys. 07 (2009) 051. 
[32] J. D. Bjorken, R. Essig, P. Schuster, and N. Toro, Phys. Rev. D 80, 075018 (2009).

[33] M. Freytsis, G. Ovanesyan, and J. Thaler, J. High Energy Phys. 01 (2010) 111.

[34] B. Batell, M. Pospelov, and A. Ritz, Phys. Rev. D 80, 095024 (2009).

[35] R. Essig, R. Harnik, J. Kaplan, and N. Toro, Phys. Rev. D 82, 113008 (2010).

[36] R. Essig, P. Schuster, N. Toro, and B. Wojtsekhowski, J. High Energy Phys. 2 (2011) 1.

[37] H. Merkel et al. (A1 Collaboration), Phys. Rev. Lett. 106, 251802 (2011).

[38] S. Abrahamyan et al. (APEX Collaboration), Phys. Rev. Lett. 107, 191804 (2011).

[39] A. Konaka et al., Phys. Rev. Lett. 57, 659 (1986).

[40] M. Davier and H. Nguyen Ngoc, Phys. Lett. B 229, 150 (1989).

[41] C. Bouchiat and P. Fayet, Phys. Lett. B, 608, 87 (2005).

[42] P. Fayet, Nucl. Phys., B187, 184 (1981).

[43] Y.-S. Tsai, Phys. Rev. D 34, 1326 (1986).

[44] C. Amsler et al. (Particle Data Group Collaboration), Phys. Lett. B 667, 1 (2008).

[45] J. Alwall, M. Herquet, F. Maltoni, O. Mattelaer, and T. Stelzer, J. High Energy Phys. 06 (2011) 128.

[46] R. Essig, P. Schuster, and N. Toro, MADGRAPH Monte Carlo for Hidden Photons in Fixed Target Experiments (unpublished).
[47] E. M. Riordan et al., Phys. Rev. Lett. 59, 755 (1987).

[48] J. D. Bjorken, S. Ecklund, W. R. Nelson, A. Abashian, C. Church, B. Lu, L.W. Mo, T. A Nunamaker, and P. Rassmann, Phys. Rev. D 38, 3375 (1988).

[49] A. Bross, M. Crisler, S. H. Pordes, J. Volk, S. Errede, and J. Wrbanek, Phys. Rev. Lett. 67, 2942 (1991).

[50] A. Hook, E. Izaguirre, and J. G. Wacker, Adv. High Energy Phys. 2011, 859762 (2011).

[51] H. Davoudiasl, H.-S. Lee, and W.J. Marciano, arXiv:1208.2973.

[52] M. Endo, K. Hamaguchi, and G. Mishima, arXiv:1209.2558.

[53] B. Aubert et al. (BABAR Collaboration), arXiv:0902.2176.

[54] B. Echenard, Mod. Phys. Lett. A 27, 1230016 (2012).

[55] J. Blumlein and J. Brunner, Phys. Lett. B 701, 155 (2011).

[56] F. Archilli et al. (KLOE-2 Collaboration), Phys. Lett. B 706, 251 (2012); S. Giovannella, J. Phys. Conf. Ser. 335, 012067 (2011).

[57] S. N. Gninenko, Phys. Rev. D 85, 055027 (2012).

[58] S. N. Gninenko, Phys. Lett. B 713, 244 (2012).

[59] T. Beranek and M. Vanderhaeghen, arXiv:1209.4561.

[60] B. Wojtsekhowski, AIP Conf. Proc. 1160, 149 (2009).

[61] The Heavy Photon Search Collaboration (HPS), https:// confluence.slac.stanford.edu/display/hpsg/.

[62] J. L. Hewett et al., arXiv:1205.2671. 